\title{
Drug Resistance Mechanism among Acinetobacter Species
}

\author{
S. Jayashree ${ }^{1}$ (D) K.G. Rajeshwari² and Mita D. Wadekar ${ }^{1 *}$ (D) \\ ${ }^{1}$ Department of Microbiology, Chamarajanagar Institute of Medical Sciences, Chamarajanagar - 571313, \\ Karnataka, India. \\ ${ }^{2}$ Aster CMI Hosital, Bangalore - 560092, Karnataka, India.
}

\begin{abstract}
Acinetobacter species cause infections that are difficult to control due to multi-drug resistance and are noted for their intrinsic resistance to antibiotics and for their ability to acquire genes encoding resistance for the production of beta-lactamases and Aminoglycoside-modifying enzymes. MBLs are molecular class B and functional group 3 beta-lactamases which have the capability of hydrolyzing all $\beta$-lactams except the Monobactam, Aztreonam. Of several MBLs, only IMP, VIM and SIM types have been detected in these species. To analyze the antibiotic resistance patterns among Acinetobacter isolates and to detect Carbapenemase and MBL among MDR Acinetobacter isolates. The descriptive study of all phenotypically identified strains and multi-drug resistant strains of Acinetobacter species was conducted. A total of $\mathbf{3 0 3}$ isolates were isolated from various samples. They were processed and identified by standard Microbiological procedures. The antibiotics susceptibility testing was performed by Kirby- Bauer disc diffusion method using CLSI guidelines. Carbapenemase production was detected by employing 3 phenotypic test methods (MHT, CDM and DDST). Of 6355 samples processed, 303 were found to be Acinetobacter species, among those $\mathbf{5 0}$ were multi-drug resistant strains. The highest isolation of MDR Acinetobacter was from endotracheal tube tip (42\%) and pus sample (32\%). Majority of MDR Acinetobacter infection was found in male patients $36(72 \%)$ compared to female patients 14 (28\%). Majority of the strains were isolated from patients $>/ 60$ years of age group (\%). A number of these isolates were more from ICU wards $(30 \%)$ followed by Surgery wards $(24 \%)$. Higher resistance for the Piperacillin/tazobactam ((82\%), followed by Ceftazidime (80\%), Imipenem (76\%) etc. and the most susceptible drug was found to be the Tigecycline (82\%) followed by Colistin (80\%). Carbapenemase production was detected by MHT and $24(48 \%)$ isolates were MHT positive. MBL production was detected by CDM and $34(68 \%)$ isolates were CDM positive and by DDST $30(60 \%)$ isolates were positive. Acinetobacter species are increasingly important nosocomial pathogens and are capable of rapid adaptation to the hospital environment. The variety of potential source of contamination or infection with these species in the hospital environment makes control of outbreaks caused by these difficult.
\end{abstract}

Keywords: Clinical samples, MBL, CDM, DDST, Antibiotic susceptibility

*Correspondence: drmdw20@gmail.com

(Received: September 01, 2020; accepted: May 05, 2021)

Citation: Jayashree S, Rajeshwari KG, Wadekar MD. Drug Resistance Mechanism among Acinetobacter Species. J Pure App/ Microbiol. 2021;15(2):883-890. doi: 10.22207/JPAM.15.2.44

(C) The Author(s) 2021. Open Access. This article is distributed under the terms of the Creative Commons Attribution 4.0 International License which permits unrestricted use, sharing, distribution, and reproduction in any medium, provided you give appropriate credit to the original author(s) and the source, provide a link to the Creative Commons license, and indicate if changes were made. 


\section{INTRODUCTION}

The genus Acinetobacter contains strictly aerobic, non-motile, Gram-negative, coccobacillary rods that are oxidase negative, nitrate negative and do not ferment sugars ${ }^{1}$. This species is the second most frequent nonfermenter encountered in clinical laboratories but with only about $1 / 10^{\text {th }}$ the frequency of Pseudomonas aeruginosa ${ }^{2}$. In humans, it has been isolated from all culturable sites. It can be part of the bacterial flora of the skin, particularly in moist regions such as the axillae, groin and toe webs, and up to $43 \%$ of healthy adults can have colonization of skin and mucous membranes with higher rates among hospital personnel and patients. Predisposing factors include the presence of a prosthesis, endotracheal intubation, intravenous catheter and prior antibiotic therapy in seriously ill patients in hospital ${ }^{3}$. The colonization of bacteria on human mucosal surface tissues is a prerequisite for an infection.The attachment of bacteria to human, animal or plant host tissues requires adhesion factors. The property of adhesion to human epithelial cells in the presence of fimbriae and / or capsular polysaccharide ${ }^{4,5}$. These species cause infections that are difficult to control due to multi-drug resistance. They are noted for their intrinsic resistance to antibiotics and for their ability to acquire genes encoding resistance for the production of beta-lactamases and Aminoglycoside-modifying enzymes ${ }^{6}$. In addition, diminished expression of outer membrane proteins, mutations in topoisomerases, and upregulation of efflux pumps also play an important part in antibiotic resistance. Common to all strains of $A$. baumannii is chromosomally encoded cephalosporinase (AmpC type). In addition to the class $C$ cephalosporinase, other $\beta$-lactamases like TEM-1 type, SHV type, CTX-M type, PER-1, and VEB-1 have been reported.7 The carbapenem resistance was associated with the overexpression of OXA-51 and OXA-23 class D carbapenemases as well as of metallo- $\beta$-lactamases (MBLs). 8 Aminoglycoside resistance is due to presence of no less than nine distinct modifying enzymes, which can be found in different combinations in some strains. Resistance to fluoroquinolones is correlated with point mutations of genes encoding DNA gyrase and topoisomerase IV.
Tetracyclines resistance has been linked with genes that encode tetracycline-specific efflux pumps.9 Outer membrane proteins (OMPs) alters bacterial membrane permeability which is associated with loss or decreased expression of porins. This group is represented by OmpA, OprD, and CarO proteins. ${ }^{10}$.

In clinical practice, Acinetobacter infections are associated closely with surgery or the use of artificial devices. The local circumstances of clinical units and their environment determine the type of infection, and the consequent risk of dissemination and an outbreak occurring. It is considered to be a low-grade pathogen, and can remain on or in the human body without causing illness ${ }^{11}$. This species can cause suppurative infections in virtually every organ system. Although it is acknowledged to be an opportunist in hospitalized patients, communityacquired infections are reported ${ }^{12}$. Multi-drug resistant strains of this isolates are a growing problem and have been widely reported and the rapid development of significant Quinolone resistance in France, Aminoglycoside resistance in Germany and Carbapenem resistance in selected regions worldwide raises an important therapeutic problem ${ }^{13}$.

\section{MATERIALS AND METHODS}

The study was undertaken at the Department of Microbiology, JSS Hospital, Mysore for a period of 6 months from July to December, 2013.

Type of study: Descriptive study Inclusion criteria

Phenotypically identified strains of Acinetobacter species isolated from different samples only are included for study.

Multidrug resistant Acinetobacter species isolated from different samples.

\section{Exclusion criteria}

Other gram negative bacilli isolated are excluded.

Various clinical samples such as urine, pus, sputum, pleural fluid, etc.., were examined for isolation and identification of Acinetobacter and antimicrobial susceptibility testing. Isolates were processed and identified by standard Microbiological procedures ${ }^{14}$. The antibiotic 
susceptibility testing was performed by KirbyBauer disc diffusion method using Clinical and Laboratory Standard Institute (CLSI) guidelines ${ }^{15}$.

The following antibiotics were tested: imipenem $(10 \mu \mathrm{g}))$, tigecycline $(15 \mu \mathrm{g})$, gentamycin $(10 \mu \mathrm{g})$, ampicillin $(10 \mu \mathrm{g})$, cotrimoxazole(23.75/1.25 $\mathrm{g})$, Piperacillin/ Tazobactum(100/10 $\mu \mathrm{g})$, ceftriaxone $(30 \mu \mathrm{g})$, ceftazidime $(10 \mu \mathrm{g})$, norfloxacin $(10 \mu \mathrm{g})$, nitrofurantoin $(300 \mu \mathrm{g})$, amikacin $(30 \mu \mathrm{g})$, levofloxacin $(5 \mu \mathrm{g})$, minocycline $(30 \mu \mathrm{g})$,

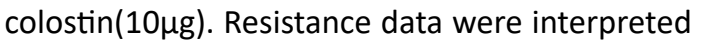
according to Clinical laboratory Standards Institute (CLSI, 2013).

\section{Detection of MBL}

Carbapenemase production was detected by employing 3 phenotypic test methods (Modified Hodge Test, Combined Disk Method and Double Disk Synergy Test).

Table 1. Total number of isolates obtained from different samples.

\begin{tabular}{lccc}
\hline Sample & $\begin{array}{c}\text { Total } \\
\text { Number }\end{array}$ & $\begin{array}{c}\text { No of } \\
\text { Acinetbacter }\end{array}$ & $\begin{array}{c}\text { MDR } \\
\text { Acinetobacter }\end{array}$ \\
\hline ET & 205 & 87 & 21 \\
Pus & 1113 & 67 & 16 \\
Urine & 2089 & 22 & 06 \\
Sputum & 1178 & 89 & 05 \\
PF & 122 & 06 & 01 \\
Blood & 1648 & 32 & 01 \\
Total & 6355 & 303 & 50 \\
\hline
\end{tabular}

\section{Acinetobacter isolates}

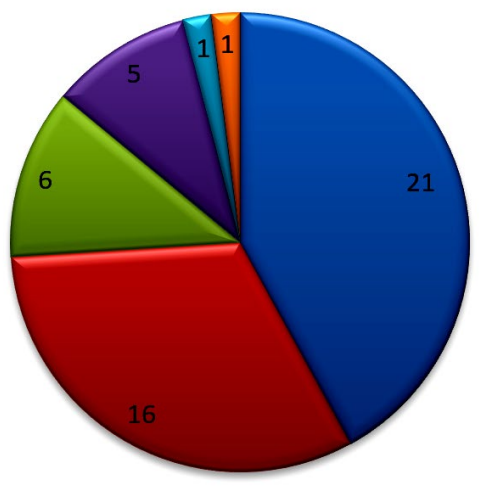

Fig. 1. Number of MDR Acinetobacter isolates isolated from various clinical Samples.

Journal of Pure and Applied Microbiology

\section{Modified Hodge Test}

Escherichia coli ATCC 25922, at a turbidity of 0.5 McFarland standards, was swabbed on the surface of Mueller Hinton agar plate, a $10 \mu \mathrm{g}$ Imipenem disc was placed at the centre and the test strain was streaked from the edge of the disc to the periphery of the plate in four directions. After overnight incubation, the plates were observed for the presence of "Clover leaf shaped" zone of inhibition. The plates with the presence of a distorted inhibition zone were interpreted as a positive result for Carbapenem hydrolysis screening.

Imipenem-EDTA Combined Disk Method

The test organisms were inoculated on Mueller-Hinton agar as recommended by the CLSI. Two $10 \mu$ Imipenem discs were placed on the plates and appropriate amount of $10 \mu$ l of EDTA solution were added to one of them to obtain the desired concentration. After $16-18 \mathrm{hrs}$ of incubation at $35^{\circ} \mathrm{C}$. if the increase in inhibition zone size was more than $7 \mathrm{~mm}$ than the Imipenem disc alone, it was considered as MBL positive.

\section{Double Disk Synergy Test}

The test inoculums was spread onto Mueller-Hinton agar. A disc of Imipenem 10 $\mu \mathrm{g}+$ sterile disc were placed on the surface of MHA, 0.5M EDTA solution were added to the sterile disc. Discs were kept at a distance between $20 \mathrm{~mm}$. The plate was incubated at $37^{\circ} \mathrm{C}$ overnight. The organisms were considered to be forming the zone Imipenem discs showed a clear-cut increase (synergy) towards the sterile disc. The increase 
occurs because the clavulanic acid inactivates the ESBL produced by the organism resulting in the formation of extended inhibitory zone.

\section{Statistical analysis}

Data analysis was done using MS Excel.

\section{Ethical considerations}

Ethical clearance was obtained from Institutional Ethical Clearance Committee of JSS Medical college, Mysore.

\section{RESULTS}

Of 6355 bacterial isolates from different clinical samples 303 (4.76\%) were found to be Acinetobacterspecies. Among the 303 of these species $50(16.5 \%)$ isolates were recognized as MDR Acinetobacter species. shown in Table 1. Highest isolation of MDR Acinetobacterwas from endotracheal tube tip (42\%) and pus sample (32\%). Other samples included urine, sputum, pleural fluid and blood samples as shown in Fig. 1. Table 2 shows that out of 50 isolates, 36 (72\%) were isolated from male patients and 14 (28\%) from female patients and the Male to female ratio was 2.5:1. As shown in Fig. 2 majority of the strains were isolated from patients $>60$ years of age group probably because of increased ICU admission in this age group and presence of other co-morbid conditions. Wardwise distribution of

Table 2. MDR Acinetobacter isolates in relation to gender

\begin{tabular}{lcc}
\hline Male & Female & Total \\
\hline 36 & 14 & 50 \\
\hline
\end{tabular}

Acinetobacterstrains were showed in Table 3 and isolates were more from ICU wards (30\%) followed by surgical wards (24\%). Table 4 shows antibiotic sensitivity pattern. The resistance pattern of these isolates showed that there was high resistance for the Piperacillin/tazobactam (82\%), followed by Ceftazidime (80\%) and Imipenem (76\%) etc. The most susceptible drug was found to be the Tigecycline(82\%) followed by Colistin (80\%). Out of 50 MDR Acinetobacter isolates, 24 (48\%) isolates were $\mathrm{MHT}$ positive. $\mathrm{MBL}$ production was detected in 34 (68\%) isolates by CDM and 30 (60\%) isolates by DDST as shown in Table 5 . and in photograhs 1 and 2 . Test results obtained by different combination of the test methods for MBL production was shown in Table 6 which shows 7 $(14 \%)$ isolates were positive by both MHT and CDM, 12 (24\%) isolates were positive by both CDM and DDST and 6 (12\%) isolates were positive by MHT and DDST. Number of Positive and Negative

Table 3. Ward wise distribution of MDR Acinetobacter isolates

\begin{tabular}{lc}
\hline Wards & Total No. of isolates \\
\hline Private ward & $02(4 \%)$ \\
Medical & $11(22 \%)$ \\
Surgical & $12(24 \%)$ \\
Pulmonology & $02(4 \%)$ \\
ICU (SICU, RICU, etc) & $15(30 \%)$ \\
Orthopaedic & $02(4 \%)$ \\
Pediatric & $02(4 \%)$ \\
NICU & $03(6 \%)$ \\
OBG & $02(4 \%)$ \\
Total & 50 \\
\hline
\end{tabular}

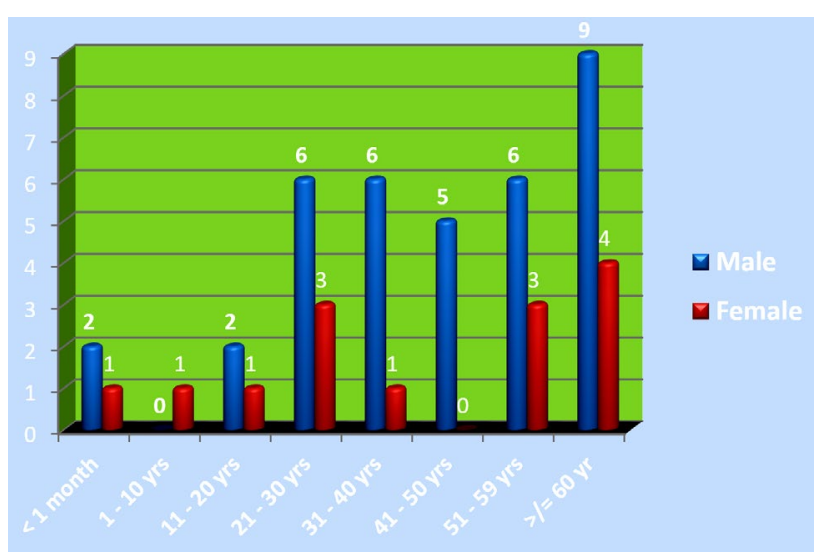

Fig. 2. Number of MDR Acinetobacter isolates in relation to Age-gender group. 
isolates by all the 3 methods is shown in table 7 which shows total number of MBL positive isolates obtained by all the 3 methods were $8(16 \%)$ and $\mathrm{MBL}$ negative isolates by all the 3 methods were found to be $03(6 \%)$.

\section{DISCUSSION}

The discovery and development of antibiotics was undoubtedly one of the greatest advances of modern Medicine. Unfortunately, the emergence of antibiotic resistance in general and also among Nonfermenters especially Acinetobacter is threatening the effectiveness of the antimicrobial agents in the treatment.

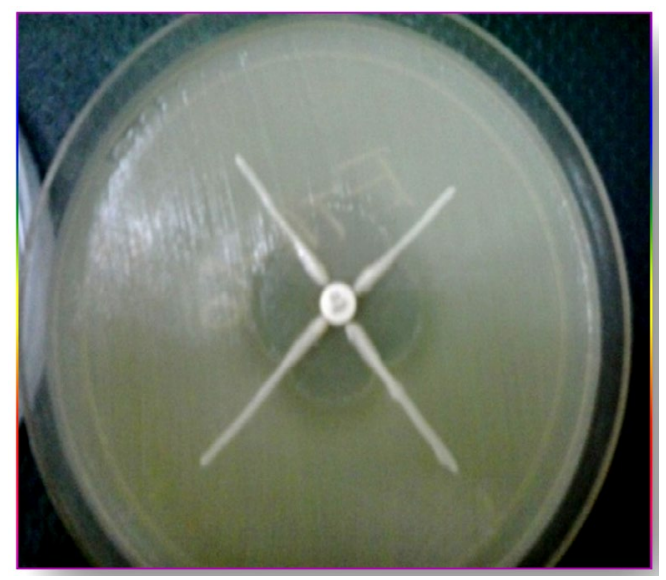

Plate 1. Mueller Hinton agar plate showing Modified hodge test positive.
To prevent cross transmission via the hands of the staff, strict hand washing policy and practice should be enforced. ${ }^{16}$ Emergence of newer microbes and ability of the bacteria to gain multidrug resistance by production of enzymes and other mechanisms has posed a serious threat in the management of nosocomial infections. Recently few Gram negative non fermentative bacilli with acquired metallo beta lactamase production have been increasingly reported in some countries.

In our study Acinetobacter species constituted $4.7 \%$ of the total bacteria isolated from different samples. Similar observations were

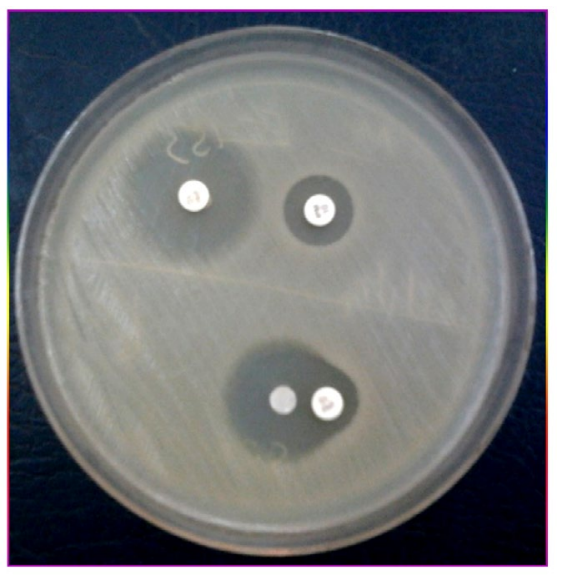

Plate 2. Mueller Hinton agar plate showing Combined disk test positive and Double disk synergy test positive

Table 4. Antibiotic sensitivity pattern of MDR Acinetobacter isolates to different antibiotics

\begin{tabular}{lccc}
\hline Antibiotics & Sensitive & $\begin{array}{c}\text { Intermediately } \\
\text { sensitive }\end{array}$ & Resistance \\
\hline Imipenem & -- & $12(24 \%)$ & $38(76 \%)$ \\
Tigecycline & $41(82 \%)$ & $09(18)$ & -- \\
Gentamycin & $09(18 \%)$ & $08(16 \%)$ & $33(66 \%)$ \\
Ampicillin & $15(30 \%)$ & $06(12 \%)$ & $29(58 \%)$ \\
Cotimoxazole & $11(22 \%)$ & $06(12 \%)$ & $30(60 \%)$ \\
Piperacillin/Tazobactum & $05(10 \%)$ & $04(8 \%)$ & $41(82 \%)$ \\
Ceftriaxone & $02(4 \%)$ & $13(26 \%)$ & $35(70 \%)$ \\
Ceftazidime & $05(10 \%)$ & $05(10 \%)$ & $40(80 \%)$ \\
Norfloxacin & $05(10 \%)$ & $10(20 \%)$ & $35(70 \%)$ \\
Nitrofurantoin & $03(6 \%)$ & $11(22 \%)$ & $36(72 \%)$ \\
Amikacin & $06(12 \%)$ & $19(38 \%)$ & $25(50 \%)$ \\
Levofloxacin & $10(20 \%)$ & $19(38 \%)$ & $21(42 \%)$ \\
Minocycline & $19(38 \%)$ & $11(22 \%)$ & $20(40 \%)$ \\
Colistin & $40(80 \%)$ & $10(20 \%)$ & -- \\
\hline
\end{tabular}


Table 5. Test Result of Carbapenemase and MBL detection by different Methods

\begin{tabular}{lccc}
\hline $\begin{array}{l}\text { Name of } \\
\text { the Test }\end{array}$ & $\begin{array}{c}\text { Total number } \\
\text { of isolates }\end{array}$ & $\begin{array}{c}\text { Number of } \\
\text { positive } \\
\text { isolates }\end{array}$ & $\begin{array}{c}\text { Number of } \\
\text { negative } \\
\text { isolates }\end{array}$ \\
\hline MHT & 50 & 24 & 26 \\
CDM & 50 & 34 & 16 \\
DDST & 50 & 30 & 20 \\
\hline
\end{tabular}

made by Rubina et al. and Hisham et al which showed $4.8 \%$ and $3.8 \%$ respectively ${ }^{17,18}$. Most of the strains were isolated from ET samples (42\%). Queenam et al isolated more MDR Acinetobacter isolates from lower respiratory tract ${ }^{19} .16 .5 \% \mathrm{MDR}$ Acinetobacter were isolated in the present study. Lt Col KK Lahiri et al studied antibiotic resistance pattern of Acinetobacter in their study and has shown multidrug resistance in 106 (69.7\%) isolates from a total of $152^{20}$. In our study out of 50 isolates, $36(72 \%)$ were isolated from male patients, and $14(28 \%)$ were from female patients. Similar gender preponderance was observed by Purti et $\mathrm{al}^{21}$. Majority of the strains were isolated from patients $>60$ years of age group. Similar findings were observed by Queenan AM and Pillar et al who isolated MDR Acinetobacter more among elderly patients $(65 \%)^{19}$.

In this study, MDR Acinetobacter were isolated more from Critical care area (30\%) followed by Surgical ward $(24 \%)$. Similar observations were made by Purti et al, and Patwardhan et al. ${ }^{19,22}$. These isolates showed increased resistance to Carbapenems, Aminoglycosides and Cephalosporins (Imipenem (76\%), Gentamicin (66\%),Ceftriaxone(70\%). Concordant findings were observed by Rubina Lone et al ${ }^{17}$ and Nazmul MHM et al. ${ }^{6}$.

In our study it was noticed that CDM was a better method for detection of MBL among the 3 methods performed. Irfan et al. in a study on Metallo- $\beta$-lactamase - producing clinical isolates of Acinetobacter species and Pseudomonas aeruginosa from intensive care unit patients of a tertiary care hospital concluded in their study that, by using imipenem-EDTA disk method (CDM) as a screening test for metallo- $\beta$-lactamase production they found a very high percentage of metallo- $\beta$-lactamase producing isolates among
Table 6. Test results obtained by different combination of the test methods for MBL production

\begin{tabular}{lcc}
\hline $\begin{array}{l}\text { Positive by } \\
\text { MHT and CDM }\end{array}$ & $\begin{array}{c}\text { Positive by } \\
\text { CDM and DDST }\end{array}$ & $\begin{array}{c}\text { Positive by } \\
\text { MHT and DDST }\end{array}$ \\
\hline 07 & 12 & 06 \\
\hline
\end{tabular}

Table 7. Number of Positive and Negative isolates by all the 3 methods

\begin{tabular}{ll}
\hline Positive by all & Negative by all \\
the 3 Methods & the 3 Methods
\end{tabular}

08

03

multidrug-resistant Acinetobacter species and Pseudomonas aeruginosa isolates ${ }^{23}$. Priyam et al conducted study on comparison of genotypic and phenotypic methods of metallo- $\beta$ - lactamase detection in Acinetobacter spp. PCR analysis of the 100 strains showed that $3(3 \%), 5(5 \%), 7$ (7\%), $26(26 \%)$, and $51(51 \%)$ strains had IMP gene, VIM gene, KPC gene, OXA gene, and NDM1 gene, respectively and also found that $\mathrm{MHT}$ on MacConkey agar (MAC) had better sensitivity when compared with the gold standard PCR than MHT on Mueller-Hinton agar (MHA). ${ }^{24}$

As PCR cannot be done in every laboratory, because of its high cost, the disc potentiation test for MBL detection should be introduced in any routine Microbiology laboratory for effective infection control and to prevent therapeutic failure. The Disc potentiation test is easy to perform, is cost effective and quite specific amongst other phenotypic methods which are used for MBL detection. The Disc potentiation test can also be done along with routine antibiotic sensitivity tests.

\section{CONCLUSION}

Acinetobacter are the "superbugs" of the modern hospital environment causing significant proportion of infections in specific patient populations, especially in critically-ill patients in the ICU's. As ubiquitous organisms (fortunately of low virulence), with few requirements for growth and survival, Acinetobacter species. are prone to persist indefinitely in the hospital environment and to cause infections periodically when iatrogenic 
factors are present, i.e., overuse of broad spectrum antibiotics and high-risk patients. Antibiotic resistance is attributed to production of extended spectrum beta-lactamase, MBL, loss of outer membrane proteins, efflux pumps and biofilm formation.

\section{ACKNOWLEDGMENTS}

The author extends gratitude to all the colleagues who helped in this study.

\section{CONFLICT OF INTEREST}

The authors declare that there is no conflict of interest.

\section{AUTHORS' CONTRIBUTION}

JS collected the statistical data, drafted the manuscript, compiled the information and designed the tables. RKG gave her valuable advice, constant guidance, moral support and inspiration in the preparation of this work. MDW supervised, supported and reviewed the manuscript and tables.

\section{FUNDING}

None.

\section{DATA AVAILABILITY}

All data analysed during this study are included in the manuscript.

\section{ETHICS STATEMENT}

Ethical clearance was obtained from Institutional Ethical Clearance Committee, India.

\section{REFERENCES}

1. Ananthanarayan and Paniker. Miscellaneous Bacteria: Acinetobacter. Textbook of Microbiology. 2013. $9^{\text {th }}$ Ed. $397-398$

2. Koneman EW, Allen SD, Janda WM, Schreckenberger PC, Winn WC et al. The Nonfermentive Gram Negative Bacteria. Colour atlas and Textbok of Diagnostic Microbiology. 2006. $6^{\text {th }}$ Ed. 353-55.

3. Manchanda V, Sanchaita S, Singh NP. Multidrug Resistant Acinetobacter. Journal of Global Infectious Diseases. 2010;2(3):291-304. doi: 10.4103/0974777X.68538

4. Jyoti R, Patil, Neelakshi R Jog. Isolation and Characterization of Acinetobacter species from upper respiratory tract of healthy humans and Demonstration of Lectin Activity. Ind J Med Microbiol. 2001;19(1):30-35.

5. Towner KJ, Collie L, Balow A, Sursman M. Acinetobacter.
Topley and Wilson's Microbiology and Microbial Infections. 1998. $9^{\text {th }}$ Ed. $1229-1235$.

6. Nazmul MHM, Jamal H, Fazlul MKK. Acinetobacter species-associated infections and their antibiotic susceptibility profiles in Malaysia. Biomed Res - India. 2012;23(4):571-575.

7. Bonomo RA, Szabo D. Mechanisms of Multidrug Resistance in Acinetobacter Species and Pseudomonas aeruginosa. Clin Infect Dis. 2006;43:S49-S56. doi: 10.1086/504477

8. Vrancianu CO, Gheorghe I, Czobor IB, Chifiriuc MC. Antibiotic Resistance Profiles, Molecular Mechanisms and Innovative Treatment Strategies of Acinetobacter baumannii. Microorganisms. 2020;8:935. doi: 10.3390/microorganisms8060935

9. Dijkshoorn L, Nemec A, Seifert H. An increasing threat in hospitals: multidrug-resistant Acinetobacter baumannii. Nat Rev Microbiol. 2007;5:939-951. doi: 10.1038/nrmicro1789

10. Kurihara MNL, de Sales RO, da Silva KE, Maciel WG, Simionatto S. Multidrug-resistant Acinetobacter baumannii outbreaks: a global problem in healthcare settings. Journal of the Brazilian Society of Tropical Medicine, 2020;53. doi: 10.1590/0037-8682-0248-2020

11. Joly-Guillou ML. Clinical impact and pathogenecity of Acinetobacter. CMI. 2005;11(11):868-873. doi: 10.1111/j.1469-0691.2005.01227.x

12. Allen D M, Hartman BJ. Mandell GL, Bennett JE, Dolin R. Acinetobacter species. Principles and practice of Infectious Diseases. 2005. $6^{\text {th }}$ Ed. 2632-2635.

13. Singla P, Sikka R, Deep A, Seema, Chaudhary U. Pattern of Antimicrobial Resistance In Clinical Isolates of Acinetobacter at a tertiary level Health care Facility in Northern India. Journal of Evolution of Medical and Dental Sciences. 2013;2(2); 160-161.

14. Collee JG, Fraser AG, Marmion BP, Simmons A. Mackie and McCartney-Practical Medical Microbiology, 14th Ed. Churchill Livingstone, New Delhi, India. 2006.

15. Clinical and Laboratory Standards Institutes (CLSI). Performance Standards for antimicrobial susceptibility testing, 29th Ed. CLSI Supplement M100. Wayne. National Committee for Clinical Laboratory Standards 2013. Pennsylvania, USA.

16. Buxton AE, Anderson RL, Werdegar D, Atlas E. Nosocomial respiratory tract infectio and colonizatio with A. calcoaceticus. Am J Med. 1978; 65: 507-513. doi: 10.1016/0002-9343(78)90777-5

17. Lone R, Shah A, Kadri SM, Lone S, Faisal S. Nosocomial Multi-Drug-Resistant Acinetobacter Infections - Clinical Findings, Risk Factors and Demographic Characteristics. Bangladesh J Med Microbiol. 2009;03(01):34-38. doi: 10.3329/bjmm.v3i1.2969

18. Ziglam H, Elahmer O, Amri S, et al. Antimicrobial resistance patterns among Acinetobacter baumannii isolated from burn intensive care unit in Tripoli, Libya. iMed Pub Journals. 2012;2(3):2.

19. Queenan AM, Pillar CM, Deane J et al. Multidrug - Resistance among Acinetobacter spp. in the USA and activity profile of key agents : results from CAPITAL surveillance 2010. Diagn Microbiol Infec Dis. 2012;73(3): 267-270. doi: 10.1016/j. 
diagmicrobio.2012.04.002

20. Lt Col Lahiri KK, Lt Col Mani NS, Lt Col Purai SS Acinetobacter spp as Nosocomial Pathogen : Clinical Significance and Antimicrobial Sensitivity. MJAFI. 2004;60:7-10. doi: 10.1016/S0377-1237(04)80148-5

21. Tripathi PC, Gajbhiye SR, Agrawal GN. Clinical and antimicrobial profile of Acinetobacter spp.: An emerging nosocomial superbug. Adv Biomed Res. 2014;3:13. doi: 10.4103/2277-9175.124642

22. Patwardhan RB, Dhakephalkar PK, Niphadkar KB, Chopade BA. A study on nosocomial pathogens in ICU with special reference to multiresistant $A$ cinetobacter baumannii harbouring multiple plasmids. Indian J Med
Res. 2008;128(2):178-187.

23. Irfan S, Zafar A, Guhar D, Ahsan T, Hassan R. Metallo - $\beta$ - lactamase - producing clinical isolates of Acinetobacter species and Pseudomonas aeruginosa from intensive care unit patients of a tertiary care hospital. Indian J Med Microbiol. 2008;26(3):243-245. doi: 10.1016/S0255-0857(21)01871-5

24. Batra P, Bajpai V, Govindaswamy A. Comparison of genotypic and phenotypic methods of Metallo - $\beta$ lactamase detection in Acinetobacter spp. J Global Infect Dis. 2020;12:141-143. doi: 10.4103/jgid. jgid_38_19 